\title{
VULNERABILIDAD SÍSMICA DE \\ QUITO ECUADOR, FASE I: CURVAS \\ DE CAPACIDAD DE LAS TIPOLOGÍAS \\ ESTRUCTURALES, PROYECTO \\ GEM - SARA
}

\section{SEISMIC VULNERABILITY OF QUITO ECUADOR, PHASE 1: CAPACITY CURVES OF STRUCTURAL TIPOLOGIES, PROJECT GEM - SARA}

\author{
CARLOS CELI ${ }^{1}$ \\ JUAN CARLOS PANTOJA ${ }^{2}$ \\ DIEGO SOSA ${ }^{3}$ \\ CARLOS AYALA ${ }^{4}$
}

Recibido: 23 de octubre de 2017

Aceptado: 31 de enero de 2018

\footnotetext{
1 Pontificia Universidad Católica del Ecuador, Facultad de Ingeniería, Quito, Ecuador (caceli@puce.edu.ec).

${ }^{2}$ Universidad San Francisco de Quito, Facultad de Ingeniería Civil, Quito, Ecuador (jcpantojam@usfq.edu.ec).

${ }^{3}$ Escuela Politécnica Nacional del Ecuador, Facultad de Ingeniería Civil, Quito, Ecuador (diego.sosa@epn.edu.ec).

${ }^{4}$ Investigador Particular, Quito, Ecuador (carlos.ayala.moya@gmail.com).
} 
||l|||| 42 


\title{
VULNERABILIDAD SÍSMICA DE \\ QUITO ECUADOR, FASE I: CURVAS \\ DE CAPACIDAD DE LAS TIPOLOGÍAS ESTRUCTURALES, PROYECTO \\ GEM - SARA
}

\section{SEISMIC VULNERABILITY OF QUITO ECUADOR, PHASE 1: CAPACITY CURVES OF STRUCTURAL TIPOLOGIES, PROJECT GEM - SARA}

\author{
Carlos Celi, Juan Carlos Pantoja, Diego Sosa, Carlos Ayala
}

Palabras clave: Tipología, No lineal-frágil, Curva de Capacidad, Costo de reposición, Escenario sísmico, mampostería.

Keywords: Typology, Non-Linear-fragile, Capacity Curve, Cost of Replacement, Seismic Scenario, Masonry.

\section{RESUMEN}

La presente investigación exhibe los resultados del análisis no lineal estático de las distintas combinaciones probables de las edificaciones multifamiliares del
Distrito Metropolitano de Quito (DMQ). Para esta investigación se identifican las 4 tipologías más recurrentes dentro de la muestra de 11556 unidades estructurales. 
Se da especial énfasis en la forma de modelar el comportamiento no lineal-frágil de elementos tipo mampostería enmarcada y no enmarcada dentro del pórtico. Del análisis se obtiene un comportamiento no lineal global de la estructura que se aproxima al comportamiento real representado por curvas de capacidad. Se someten las distintas curvas de capacidad de cada modelo teórico a dos escenarios sísmicos (100 y 475 años de período de retorno), conside- rando la amplificación sísmica debido al tipo de suelo B - C presentes en la NEC15. Se realiza este análisis para obtener un primer vistazo del costo de reposición ante el colapso de las estructuras examinadas en los escenarios sísmicos propuestos, y que serán validados en la posterior investigación denominada "Fragility and Vulnerability Curves of Structural Typologies, Project GEM-SARA)".

\section{ABSTRACT}

The present investigation shows the results of the static non-linear analysis of the different probable combinations of the multifamily buildings of the Metropolitan District of Quito (DMQ). For this research, are identified the four most recurrent structural typologies within the sample of 11556 structural units.

Special emphasis is given to the way of modeling the nonlinear-fragile behavior of framed and non-framed masonry elements. From the analysis, a global non-linear behavior of the structure is obtained that approximates the real behavior represented by capacity cur- ves. The different capacity curves of each theoretical model are subjected to two seismic scenarios (100 and 475 years of return period), considering the seismic amplification due to the type of soil B - C present in the NEC15.

This analysis is carried out to obtain a first glance of the cost of replacement before the collapse of the structures examined for the proposed seismic scenarios, and that will be validated in the later research called "Fragility and Vulnerability Curves of Structural Typologies, Project GEM-SARA)". 


\section{INTRODUCCIÓN}

\section{TIPOLOGÍAS RESIDENCIALES DEL DMQ}

A finales del año 2015, se inicia el proceso de levantamiento de información de la configuración geométrica-estructural, de una muestra de 11556 edificaciones multifamiliares de la ciudad de Quito. Para este fin se realiza un convenio con Global Earthquake Model (GEM), para emplear la metodología que esta institución emplea en el análisis de vulnerabilidad sísmica de otras ciudades piloto alrededor del mundo.
Para ello previamente se realiza un análisis de zonificación del $\mathrm{DMQ}$, con base en la información socio-económica y un primer muestreo geométrico estructural; con base en estos datos se clasifica al DMQ en zonas homogéneas, en las cuales se realiza la distribución para el muestreo de 11556 edificaciones como muestra representativa de las estructuras multifamiliares hasta 4 pisos.

En la Figura 1 se puede observar el mapa DMQ dividido en zonas homogéneas y la localización del muestreo de las 11556 edificaciones.

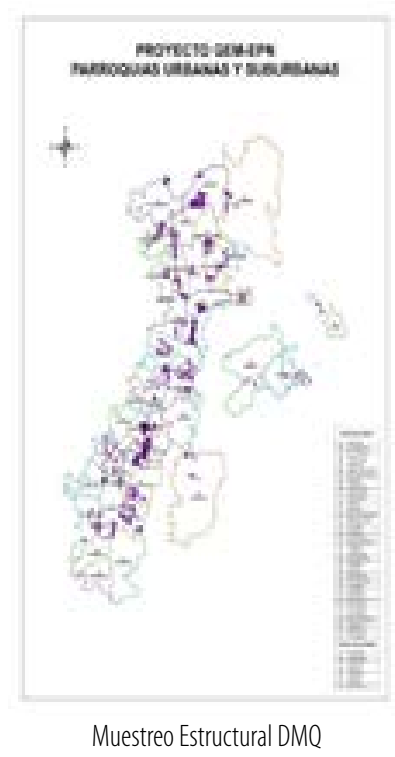

Figura 1. DMQ, Zonas Homogéneas/ Muestreo Estructural. 
Para el inventario geométrico estructural de las edificaciones del DMQ, se emplea la aplicación (IDCT do survey) desarrollada por GEM. La aplicación posee más de 40 item de entrada de datos, de los cuales destacan:

- Inventario del sistema resistente a carga lateral
- Inventario de materiales constitutivos estructurales y no estructurales - Georeferenciación, etc.

De la información recolectada se identifican las 4 tipologías recurrentes en la muestra, las cuales se detallan a continuación.

\section{TIPOLOGÍA 1}

Comprende edificaciones de un piso con techo de zinc, eternit o losa de hormigón. Se definen las dimensiones entre ejes de tres metros como aproximación al rango de 2.40 a 3.20 metros

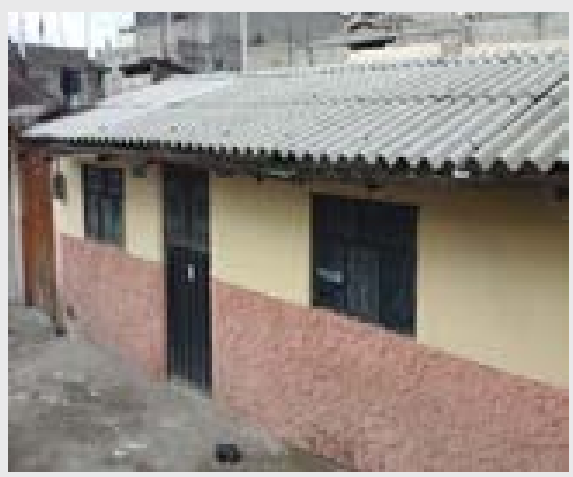

obtenidos en campo. Estas dimensiones se basan en contabilizar el número de bloques entre columnas (entre 6 y 8 unidades) y una longitud de bloque de hormigón de 0.40 metros, este procedimiento se emplea en el resto de tipologías.

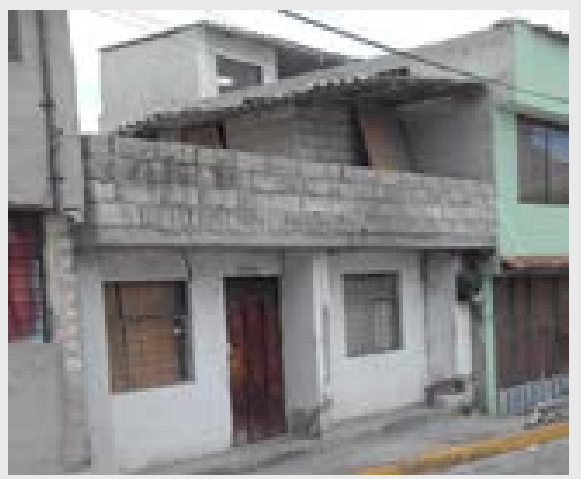

Figura 2. Fotografía aleatoria (Muestra de la Tipología 1). 


\section{TIPOLOGÍA 2}

Comprende edificaciones de dos tipo "L"y entrepisos de hormigón.

a tres pisos, con irregularidad en planta
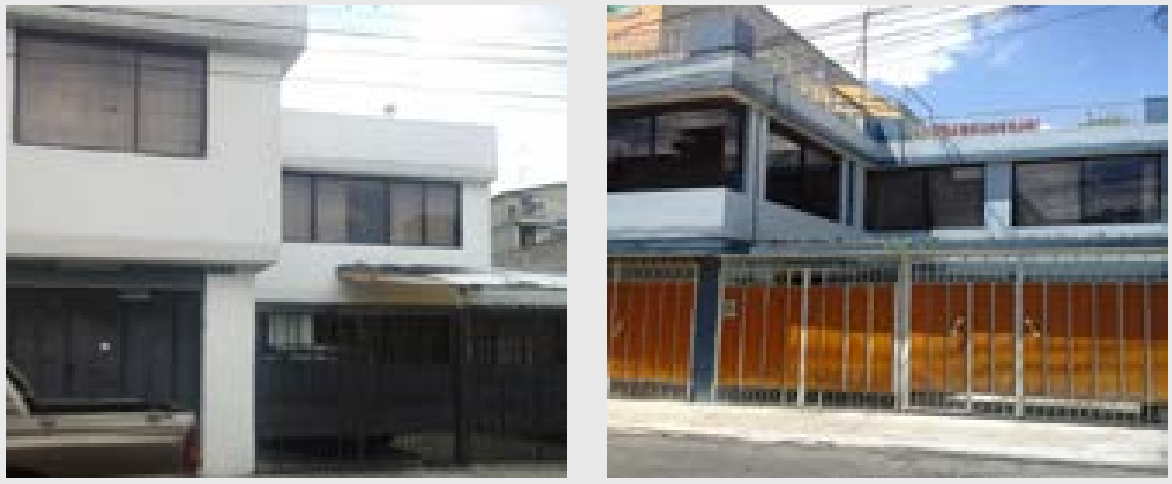

Figura 3. Fotografía aleatoria (Muestra de la Tipología 2).

\section{TIPOLOGÍA 3}

Comprende edificaciones de hormigón. dos a tres pisos que tienen una forma
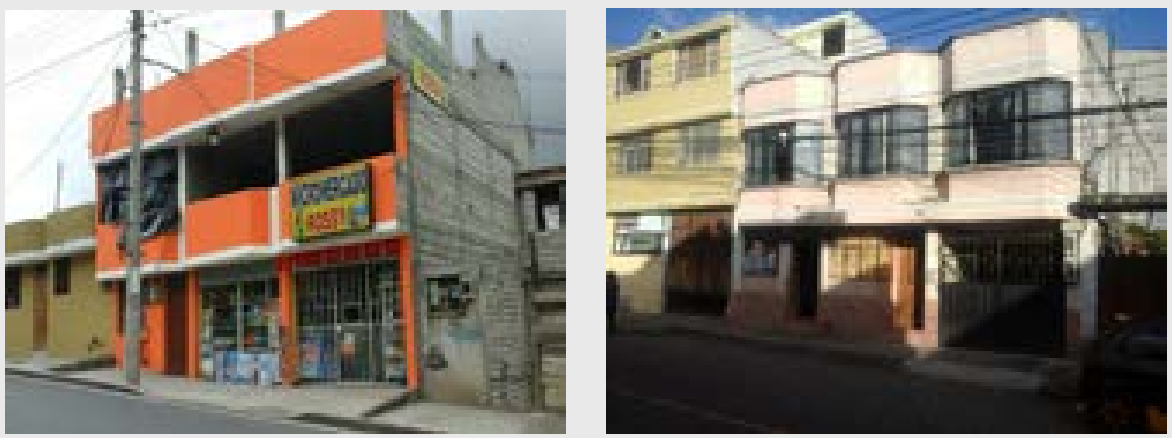

Figura 4. Fotografía aleatoria (Muestra de la Tipología 3). 


\section{TIPOLOGÍA 4}

Comprende edificaciones de cuatro a seis pisos, que tienen una forma en
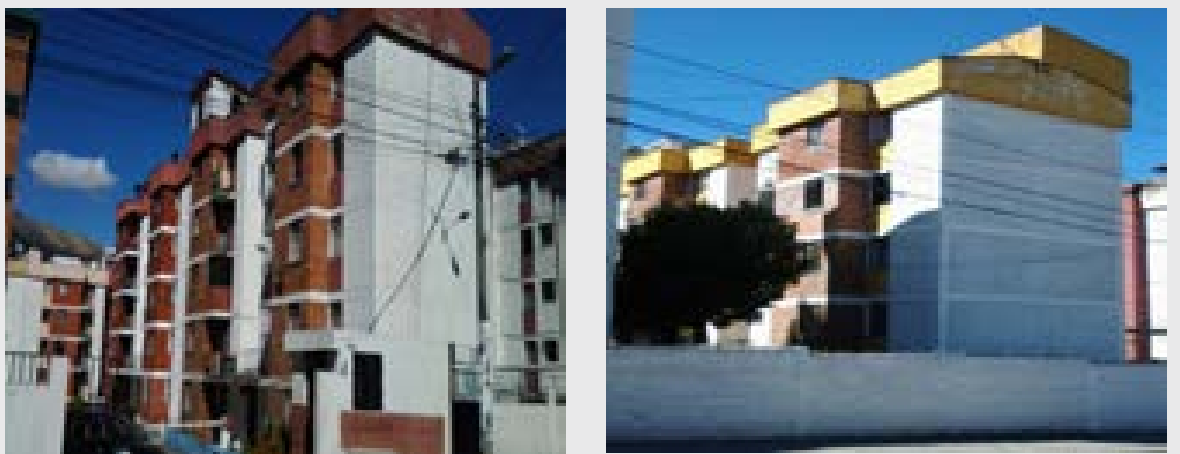

Figura 5. Fotografía aleatoria (Muestra de la Tipología 4).

De la muestra de 11556 unidades critas representan el $88 \%$ de la totalidad. de edificaciones residenciales y mixtas En la Figura 6 se observa la distribución uni y multifamiliares, las tipologías des- de las tipologías dentro de la muestra.

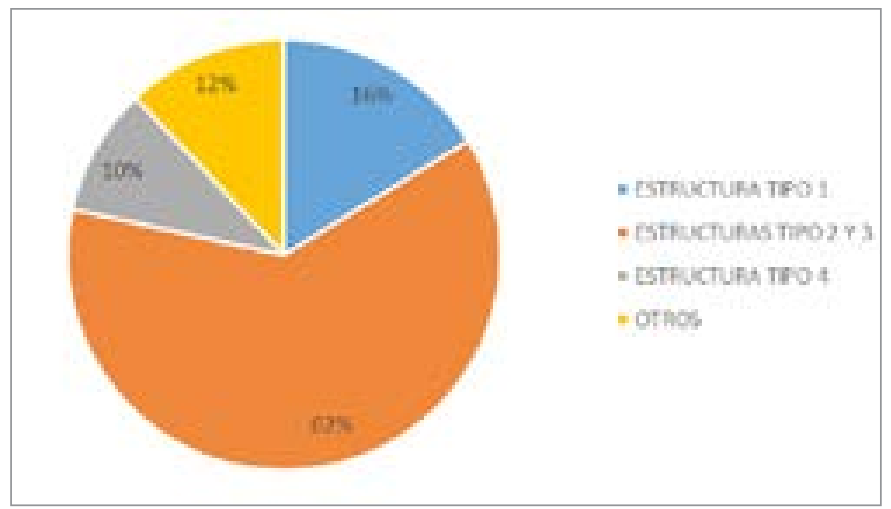

Figura 6. Distribución de la Muestra. 
Puesto que la modelación matemática debe representar la variabilidad de materiales, distancia entre ejes, número de pisos, distribución de mamposterías enmarcadas y no enmarcadas en la estructura tridimensional; se decidió realizar 114 modelaciones matemáticas no lineales distribuidas en las 4 tipologías mencionadas. Las combinaciones por distintos materiales, secciones, pisos etc., serán presentados en el artículo sobre el modelo de exposición.
No obstante cabe recalcar que de las 114 modelaciones, para este artículo se presenta el resultado de 60 modelos matemáticos; en la versión final de este artículo se presentarán los resultados totales.

La representación no lineal de cada tipología, se realiza a través de 4 distintos modelos matemáticos; las cuales se describen a continuación.

\section{CURVAS DE CAPACIDAD}

MODELO DE FIBRAS (TIPOLOGÍA 1)

Para la obtención de las curvas de capacidad mediante el método de fibras, se utiliza el programa de análisis no lineal SeismoStruct [1]. Dicho programa se basa en el método de fibras el cual considera que el comportamiento inelástico se desarrolla a lo largo de todo el elemento; para lo cual se utilizan secciones de integración transversales, de tipo Gauss-Lobatto, las mismas que están discretizadas en fibras.
Las fibras de forma individual obedecen a un comportamiento esfuerzo-deformación uniaxial; el estado tensional y deformacional de cada sección se lo obtiene de la integración de la respuesta esfuerzo-deformación uniaxial no lineal de cada una de las fibras. Para el caso de elementos de hormigón armado se tienen tres materiales constitutivos: hormigón no confinado, hormigón confinado y acero de refuerzo. 


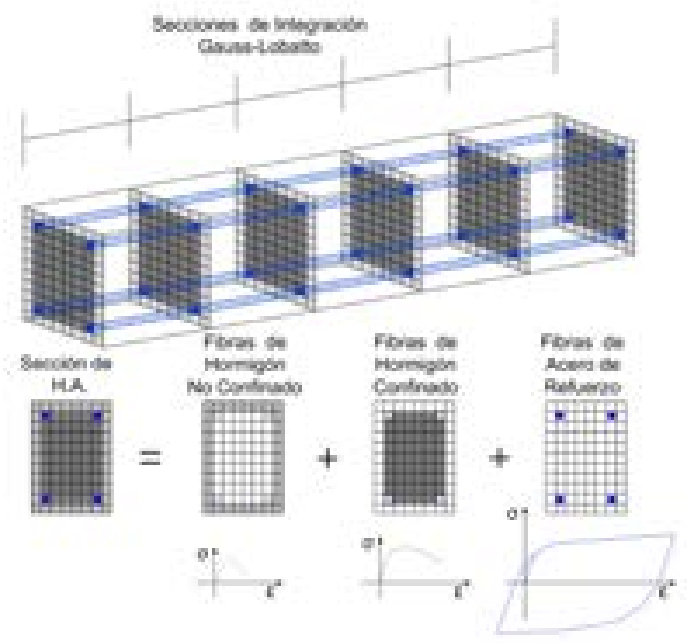

Figura 7. Representación del comportamiento no lineal de la sección transversal. Aproximación por fibras.

Es relevante considerar el efecto de la mampostería de relleno, para lo cual en el programa SeismoStruct se emplea un panel de relleno, cuyo modelo analítico obedece al propuesto por Cri- safulli [2], e implementado por Blandon [3]; además para paneles que presentaban aberturas como ventanas y puertas, se toma el criterio de Smyrou [4].
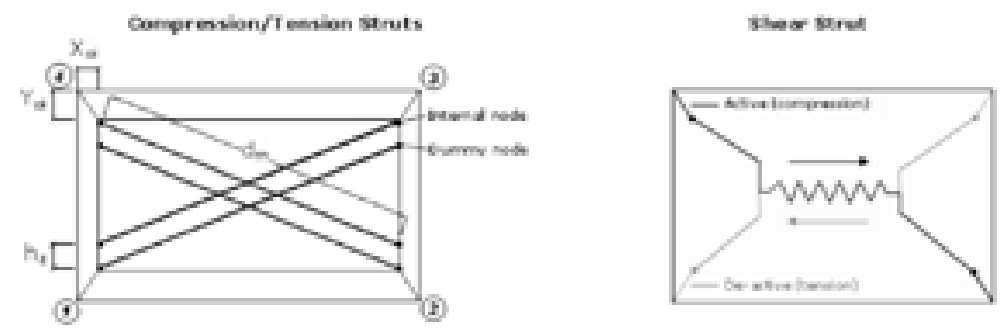

Figura 8. Panel relleno inelástico. Elemento tipo 1. 
La tipología 1 analizada por esta y mampostería de bloque. En la Figura 9 metodología, presenta un sistema resis- se presenta a un esquema de la tipología tente a carga lateral de tipo aporticado estudiada.

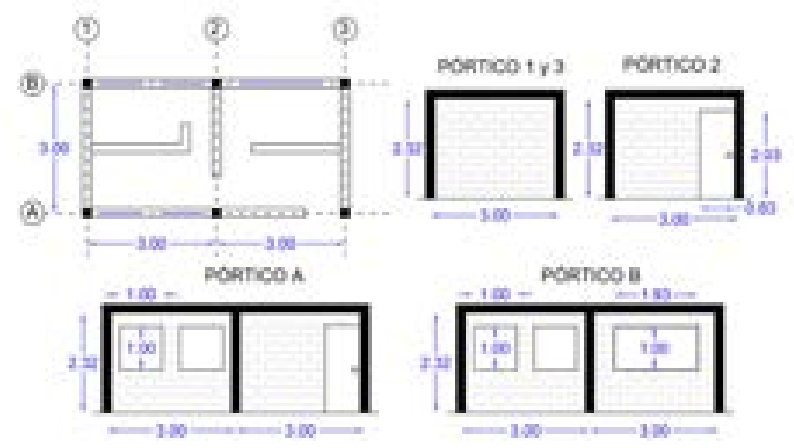

Figura 9. Tipología de un piso.

De Alomoto [5] se obtienen los el modelo de mampostería enmarcada. resultados experimentales del ensayo En la Figura 10 se presenta el resultado de pórticos con mampostería típica experimental versus la aproximación Ecuatoriana, mediante el cual se calibra matemática.

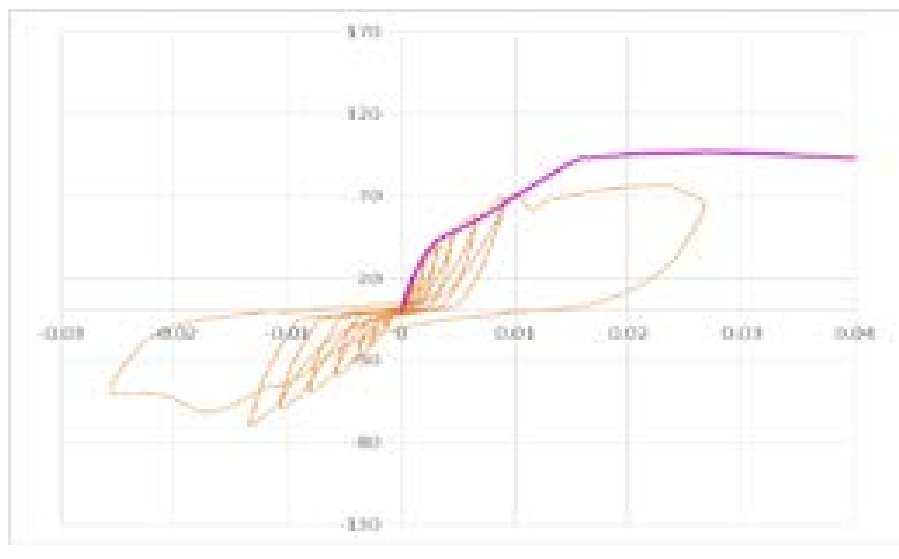

Figura 10. Ensayo cíclico experimental y curva envolvente analítica (KN-m). 
Una vez que se calibra el modelo de mampostería enmarcada, se realiza combinaciones (Globales) lineales de los pórticos en un mismo sentido, considerando los diferentes tipos de materiales.

\section{Tabla 1. Combinaciones, Curvas de Capacidad.}

\begin{tabular}{ccc}
\hline Nombre & Combinación & Materiales \\
\hline Combo 1 & Pórtico A + Pórtico B & Buenos \\
Combo 2 & Pórtico A + Pórtico B & Promedio \\
Combo 3 & Pórtico A + Pórtico B & Malos \\
Comb0 4 & Pórtico 1 + Pórtico 2 + Pórtico 3 & Buenos \\
Comb0 5 & Pórtico 1 + Pórtico 2 + Pórtico 3 & Promedio \\
Comb0 6 & Pórtico 1 + Pórtico 2 + Pórtico 3 & Malos
\end{tabular}

\section{MODELO DE RIGIDEZ EQUIVALENTE DE MUROS (TIPOLOGÍA 2)}

El proceso de modelación se realiza considerando la variación de la mampostería en la rigidez lateral del pórtico. Se toman en cuenta cuatro configuraciones de muros dentro del pórtico, las mismas que se observan en la Figura 11. Existe una variación en la rigidez que aporta la mampostería según su porcentaje aberturas y su ubicación dentro del muro; para describir la curva cortante-desplazamiento en la cabeza del muro se emplean dos procedimientos. El procedimiento descrito por Reinhorn [15] se usa para las configuraciones de muros 1 y 2; el procedimiento descrito por Asteris [16] se emplea para las configuraciones 3 y 4.

En la modelación del comportamiento se considera la relación de as- pecto del muro, la capacidad a flexión de las vigas y columnas en el nudo, la sobrecarga de compresión del muro, la ubicación y relación de aspecto de la abertura, la pérdida de resistencia por compresión, entre otras. Estas variables modifican la resistencia y dirección del puntal a compresión del sistema. En la Figura 12 se muestran las curvas cortante-desplazamiento en la cabeza del muro para los materiales promedio.

Las vigas del pórtico tienen una sección de $0.30 \times 0.20 \mathrm{~m}$ y las columnas de $0.30 \times 0.30$, con cuantías de $1.3 \%$ y $1.0 \%$ respectivamente. La modelación de elementos que conforman el pórtico se realiza mediante rótulas plásticas de tipo momento-rotación en los extremos de vigas y columnas. Para la definición de las rotulas de vigas, se calculan diagramas momento-curvatura de las 
secciones mediante un modelo de fibras que contempla el concreto no confina-

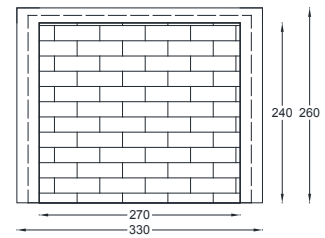

(a)

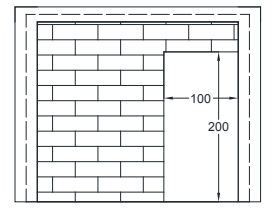

(b)

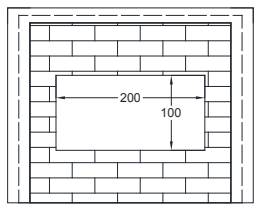

(c)

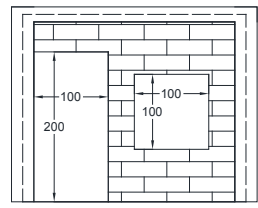

(d)

Figura 11. Marco plano con paneles rellenos, a). Configuración n. ${ }^{\circ} 1$. b). Configuración n. ${ }^{0} 2$. c). Configuración n. ${ }^{\circ}$ 3. d). Configuración n. ${ }^{4}$. Unidades en mm.

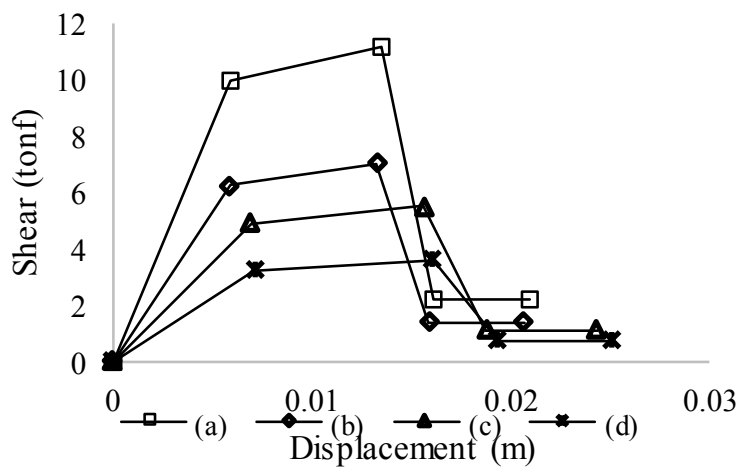

Figura 12. Cortante versus Desplazamiento en paneles rellenos con mampostería. a). Configuración n. ${ }^{\circ}$ 1. b). Configuración n. ${ }^{\circ}$ 2. c). Configuración n. ${ }^{\circ}$ 3. d). Configuración n. ${ }^{0}$ 4. Unidades en mm.

Para la definición de la rótula se considera que la sección llega a la falla cuando la deformación unitaria del concreto confinado $\varepsilon_{c}$ alcanza un valor de 0.005 a compresión. Un proceso similar se realiza para modelar las columnas, sin embargo, se considera que la capacidad a momento de un elemento depende de la carga axial que soporta.

En la Figura 13 se muestra la idealización matemática de la rótula plástica en la columna 0.30×0.20 m además se indican las curvaturas para las cuales se consideran los niveles de desempeño IO, LS y CP. 


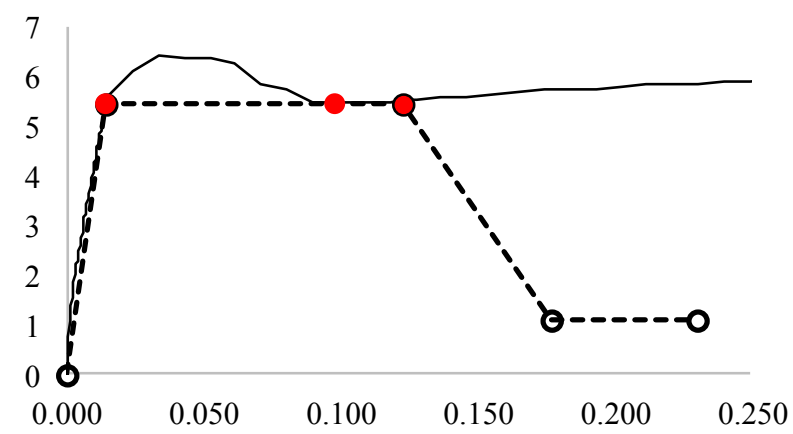

Figura 13. Momento curvature. Sección CL0.30x0.20 m. Unidades ton y 1/m.

Para la modelación estructural cada configuración de mampostería, se se emplea el software de análisis no emplea un elemento propio del software lineal PERFORM 3D [17]. Para las vigas para muros que permite controlar el se emplean rótulas plásticas momento- desplazamiento según el cortante en la curvatura. Para las columnas, se emplean rótulas plásticas momento-curvatura con interacción carga axial-momento. Este elemento incluido en el software, permite que el momento de fluencia $\left(M_{y}\right)$ de la columna varíe según la carga axial del elemento. Para la modelación de cabeza del muro.

De los ensayos experimentales obtenidos por Alomoto [5] de un pórtico con mampostería típica se calibran las cuatro configuraciones de mampostería. En la Figura 14 se observa la calibración del modelo final. 


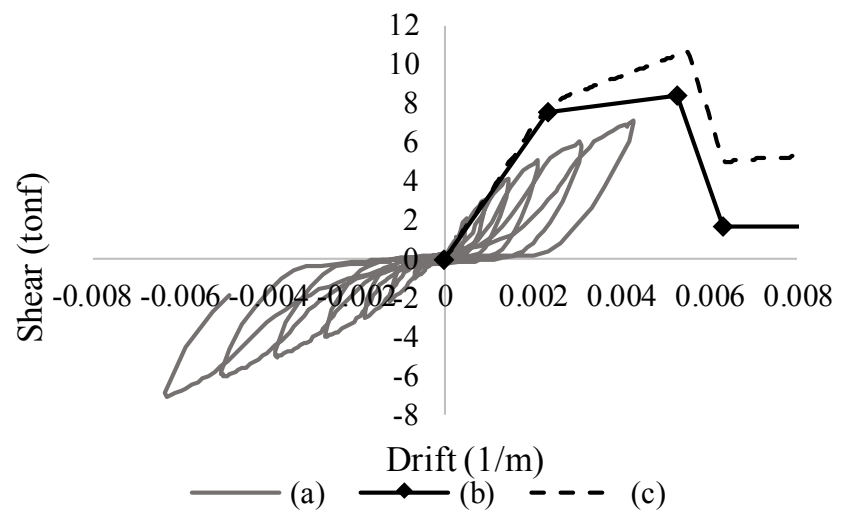

Figura 14. a). Prueba cíclica experimental. b). Reinhorn et al [6] modelo de panel relleno. c). Respuesta lateral del marco plano relleno.

Se realiza una modelación matemática tridimensional considerando varias distribuciones probables de mampostería de la tipología 2. Los análisis se realizan para la misma configuración en planta variando la altura del edificio desde 2 a 4 pisos. Se realizan además variaciones sobre las propiedades mecánicas para cada piso en los materiales constructivos.

\section{MODELO PLASTIFICACIÓN CONCENTRADA (TIPOLOGÍA 3)}

Para la modelación de la tipología 3, se realiza la representación de los mecanismos de disipación de energía mediante plastificación concentrada.
Para ello se comete un análisis lineal controlado por derivas inelásticas; con el objetivo de determinar la forma de la fuerza lateral resultante del análisis modal, de manera que se pueda obtener el patrón de carga incremental; además es necesario establecer el diagrama de momentos ante carga lateral de los distintos elementos estructurales resultantes en primera instancia del análisis lineal controlado por derivas inelásticas.

Se calculan las curvas constitutivas de los materiales, para Hormigón mediante Jirsa[9] - Papovic[10] - Mander[11] y Acero con base en el modelo trilineal de Opesees [12]. 


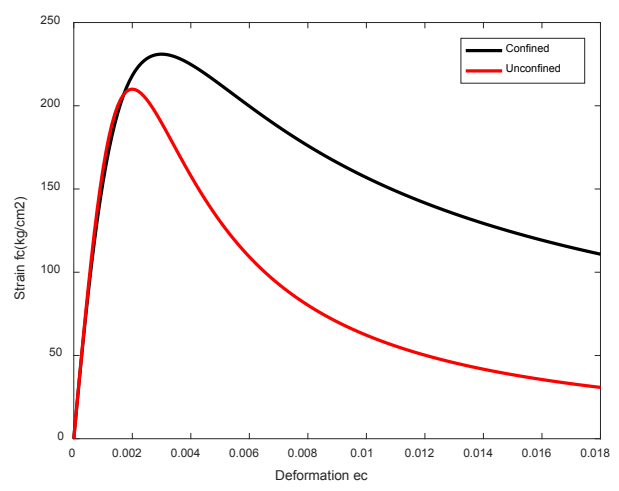

Figura 15. Curva esfuerzo - deformación para hormigón confinado y no confinado, k (Mander) =1.1.

Con base en las curvas constitutivas de los materiales, se elaboran los distintos diagramas momento-curvatura para cada tipo de sección y armado; en la Figura 16 se presenta uno de los diagramas Momento curvatura de una de las distintas configuraciones de secciones.
Los diagramas momento-curvatura se obtienen mediante programación en Matlab [13] por compatibilidad de deformaciones, método AYU (dovelas) y SAP2000 [12] (section designer).

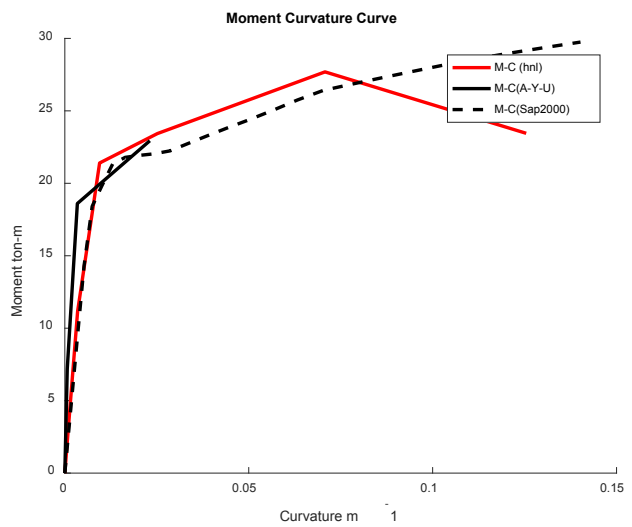

Figura 16. Diagrama momento curvatura, col $30 \times 30$. (Ton-m). Tipología 4. 
Los diagramas momento - curvatura se limitan cuando el bloque de compresión no es capaz de generar una fuerza de al menos un $30 \%$ de la fuerza de total de compresión, también se implementa un control de estabilidad física dentro de la solución del estado tensional; es decir, si la profundidad del bloque de compresión es menor que el recubrimiento de la sección de análisis, se considera una falla frágil en el elemento.

En la Figura 16a, se observa la implementación de los criterios antes mencionados para la determinación de la ductilidad final por curvatura.

Para determinar la longitud de plastificación de cada elemento se busca el momento de fluencia (My) del diagrama momento curvatura en los dia- gramas de momentos presente en los pórticos debido a carga lateral analizada linealmente. Una vez establecida la longitud de plastificación de cada mecanismo de disipación de energía del pórtico, se procede a calcular el centro de gravedad de los mismos para la posterior implementación del momento rotación correspondientes derivados de los distintos diagramas momento curvatura.

En la Figura 16b, se presenta de manera sintetizada el proceso de cálculo empleado para la estimación capacidad plástica por rotación de los distintos elementos estructurales, con base en el procedimiento descrito en ASCE 41 [9]; considerando la corrección por ductilidad final, previamente descrito.

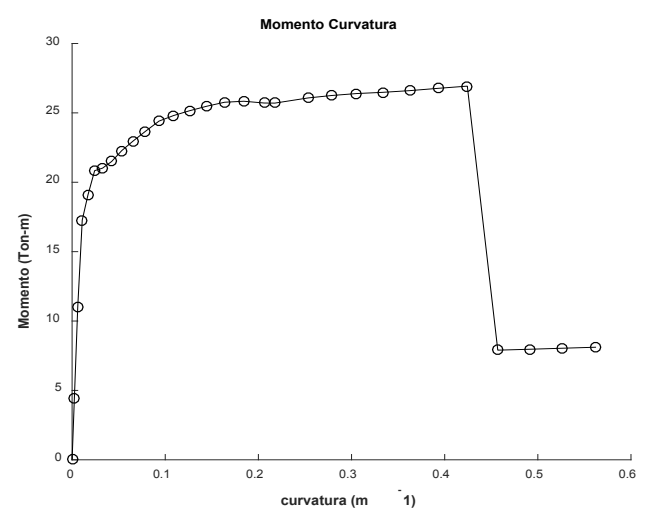

Momento Curvatura no corregido, colum aleatoria, Tipología 3 


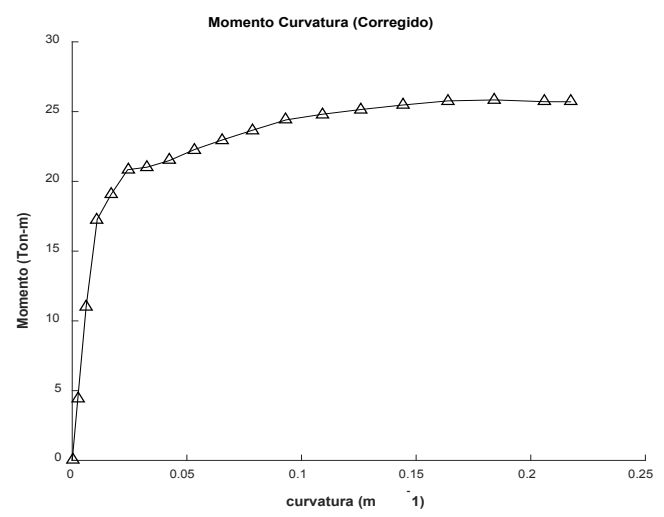

Momento Curvatura corregido, colum aleatoria, Tipología 3

Figura 16a. Diagrama momento curvatura, columna aleatoria Tipología 4. Corregido y no corregido.

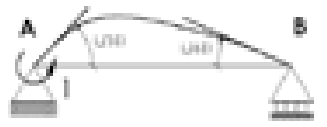

$M_{3}=1$
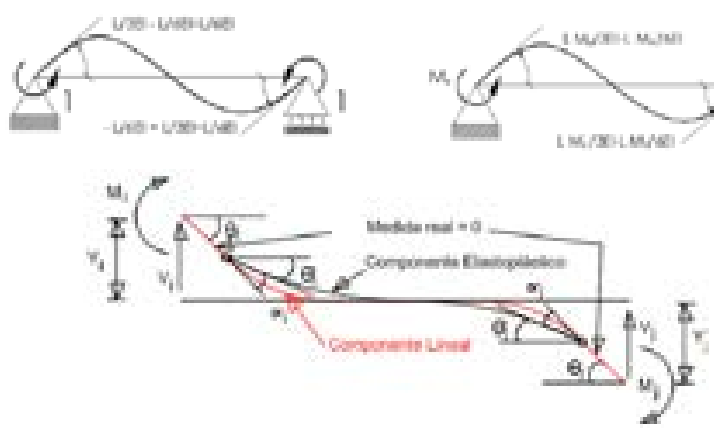

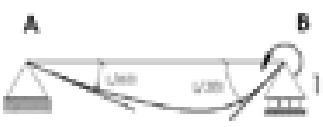

$\mathrm{Mb}=1$

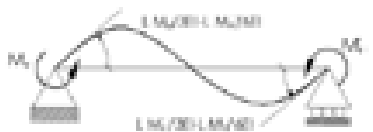

Figura 16b. Proceso lógico de generación de capacidad plástica y ductilidad por rotación.

Se consideró en la generación de los distintos diagramas momento rotación además del proceso antes expuesto, la metodología presente en ASCE 41
[21]; puesto que en ciertos casos de la presente investigación el momento rotación producto de este procedimiento envuelve de mejor manera la capacidad 
plástica representada en la curva de capacidad. El proceso de ASCE 41 se empleó en las secciones que presentaban un confinamiento transversal relativamente bueno $(k>1.1)$.

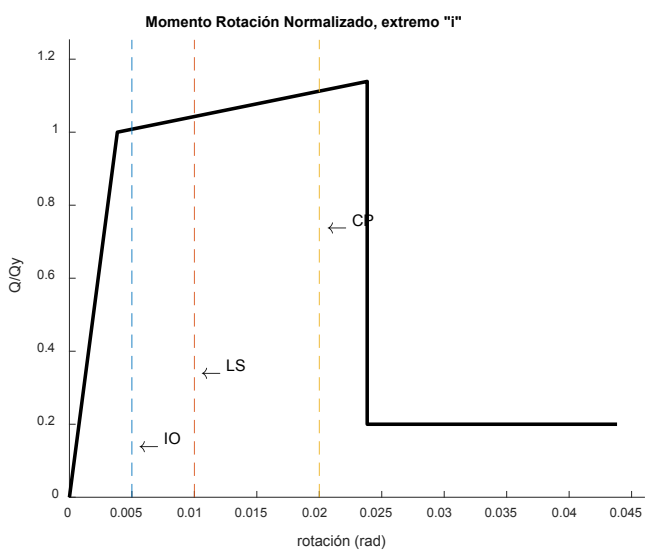

Momento Rotación corregido, colum aleatoria, Tipología 3. Metodología integración $\mathrm{M}-\mu$

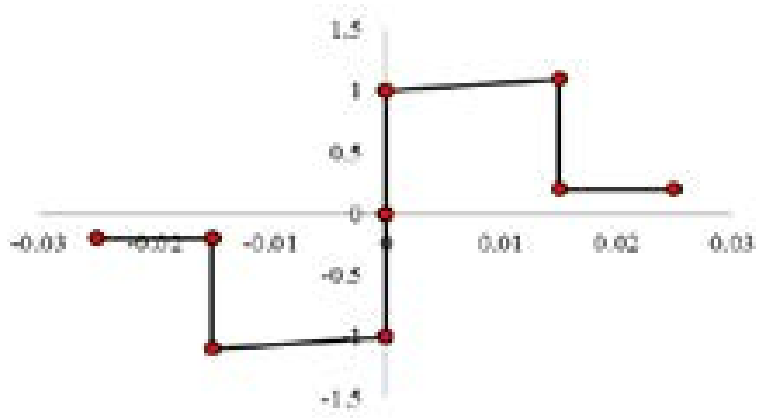

Momento Rotación (back bone) corregido, colum aleatoria, Tipología 3. Metodología ASCE 41

Figura 16c. Back Bone, Momento Rotación, sección aleatoria, as = 1.3 \%, Typology 3, modelo 16. 
El modelo matemático que representa el comportamiento no lineal-frágil de la mampostería, se realiza mediante la generación de un puntal de compresión, que simula el comportamiento de la mampostería enmarcada entre pórticos. El modelo matemático del puntal de compresión se ejecuta mediante un link no lineal ubicado a la mitad de la longitud del elemento que posee una rigidez inicial lineal, limitada por una falla frágil al alcanzar la capacidad máxima a compresión; la cual es controlada por el desplazamiento medido en laboratorio. Para ello se emplearon los resultados experimentales realizados por Alomoto [5].

La capacidad máxima y ancho equivalente del puntal se calibran mediante varios modelos cuyos resultados se aproximan al ensayo realizado por Alomoto [5]. El modelo matemático del puntal de compresión no lineal - frágil cambia según la distancia entre ejes de cada modelo describiendo la variabilidad de la tipología 3.
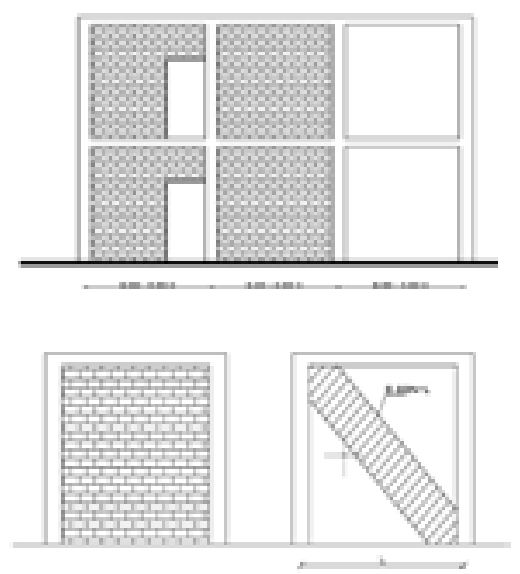

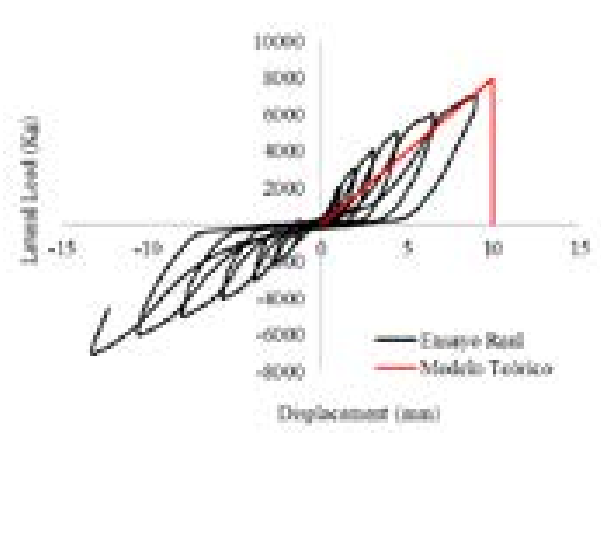

Figura 17. Modelo Experimental versus Modelo Matemático, Tipología 3, Modelo 17.

\section{MODELO CELOSÍA (TIPOLOGÍA 4)}

La representación matemática de la tipología 4 se la realiza mediante
Opensees [18]; el comportamiento de todo el pórtico, incluida la mampostería, se puede representar como un ensam- 
blaje de celdas de celosía, en el que cada celda se conforma de dos elementos
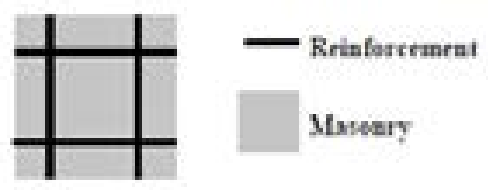

verticales, dos elementos horizontales y dos diagonales.

Figura 18. Modelo tipo "Celosia"

El procedimiento para asignar elementos de celosía es una modificación de la metodología propuesta por Panagiotou [18]; y lo propuesto por Williams [19] para mamposterías enmarcadas y no enmarcadas. Se emplea lo propuesto por Ayala [20], para la modelación de mampostería armada con relleno parcial.

\section{MODELO CELOSÍA NO LINEAL}

Los elementos de borde son definidos como la sección transversal de la

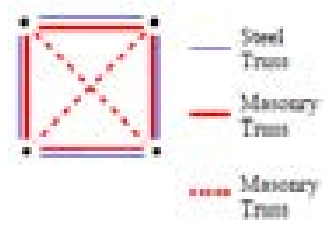

columna o viga que rodea a la mampostería. Los elementos interiores de mampostería se dividen equitativamente en cuatro partes tanto vertical como horizontalmente. Los elementos diagonales que también son de mampostería y que unen los elementos verticales y horizontales, se definen con un espesor igual a la mampostería y con ancho efectivo beff. Figura 19.

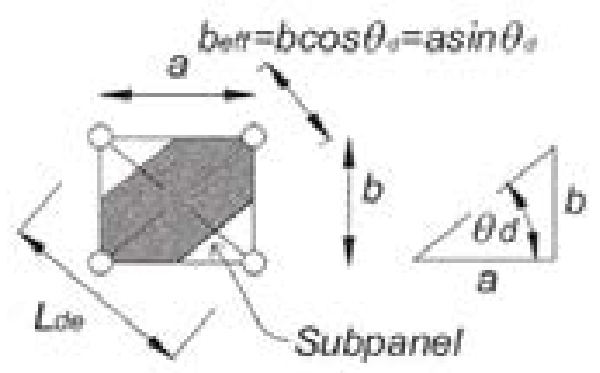

Figura 19. Ancho efectivo del elemento diagonal "celosia". 
Cada uno de los elementos de la celosía sigue el comportamiento esfuerzo-deformación uniaxial. Para los elementos de mampostería diagonal, se emplea el material ConcretewBeta.

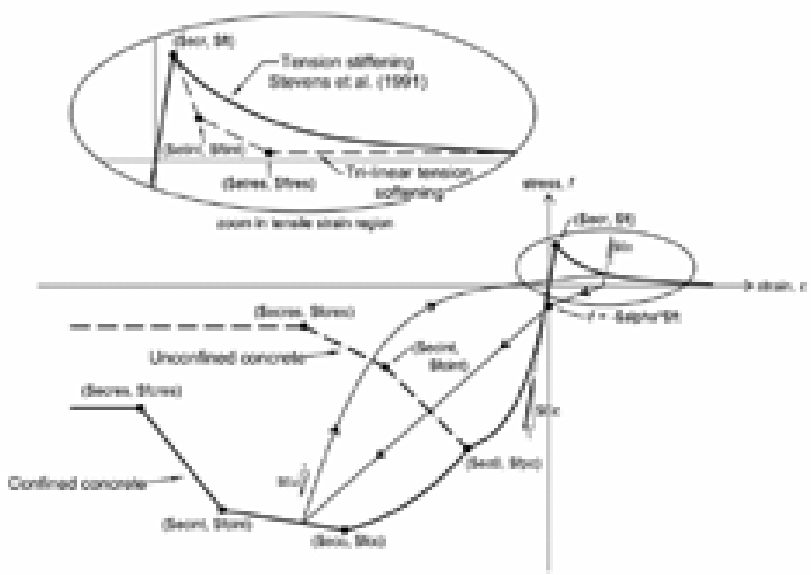

Figura 20. Material Uniaxial. ConcretewBeta

\section{RESULTADOS}

En resumen, se emplean dos distintas filosofías de análisis para la simulación matemática de la plastificación de los elementos marco plano; es decir plastificación concentrada y plastificación distribuida. Mientras que para la representación matemática del comportamiento no lineal-frágil de la mampostería enmarcada y no enmarcada, se realiza con base en 4 distintas formas de modelación fundamentadas en: Punta-
La envolvente de la curva esfuerzo-deformación se basa en el modelo Fuji Hoshikuma. 
figuración y distribución de rigidez que se observan en las Tipologías Quiteñas.

\section{CURVAS DE CAPACIDAD}

La técnica empleada para obtener las distintas curvas de capacidad, indiferentemente de la tipología es la técnica Non linear Static PushOver (NSP). En primera instancia se realiza el cálculo hasta encontrar la estabilidad numérica para cada uno de los distintos modelos matemáticos. Debido a que las tipologías son de baja altura, la forma de la carga incremental lateral sigue una distribución triangular. La forma modal de distribución de carga lateral no representa un cambio significativo en la respuesta estructural.

En primera instancia se realiza el cálculo hasta encontrar la estabilidad numérica para cada uno de los distintos modelos matemáticos.

Debido a que las tipologías son de baja altura, la forma de la carga incremental lateral sigue una distribución triangular; es decir la forma modal de distribución de carga lateral no representa un cambio significativo en la respuesta estructural. Con base en las distintas técnicas de modelación matemática descritas anteriormente se obtuvieron las siguientes curvas de capacidad hasta estabilidad numérica.

Es importante señalar que el objetivo final es generar la fragilidad intrínseca de cada tipología y ya que la función de desviación estándar acumulada y el valor medio de desplazamiento espectral necesarios para el cálculo de fragilidad, son altamente susceptibles al valor desplazamiento teórico de colapso d; dicho punto se calcula mediante un control de fragilidad local dentro del análisis NSP el cual para la presente investigación resulta menor que el valor generado en la estabilidad numérica dentro del análisis NSP.

El efecto se observa para el cálculo de la fragilidad estructural ya que se sobreestima la ductilidad del sistema, lo que genera una estimación incorrecta de la probabilidad de alcanzar un umbral de daño.

En la Figura 21, se observa las curvas de capacidad de los modelos matemáticos que representan la variabilidad de la tipología 1. 


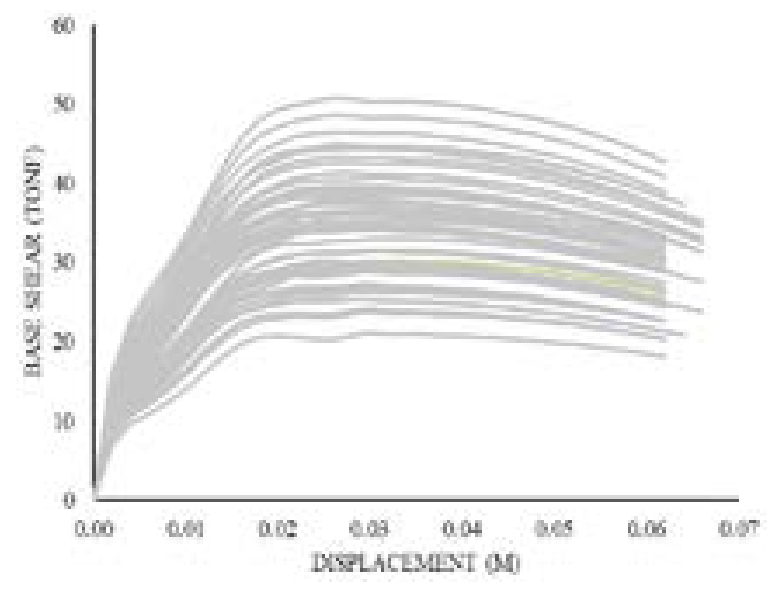

Figura 21. Curvas de Capacidad, Tipología 1, Modelos 1 - 47. Corregidas por fragilidad local.

En la Figura 22, se observan las matemáticos que representan la variabicurvas de capacidad de los modelos lidad de la tipología 2.

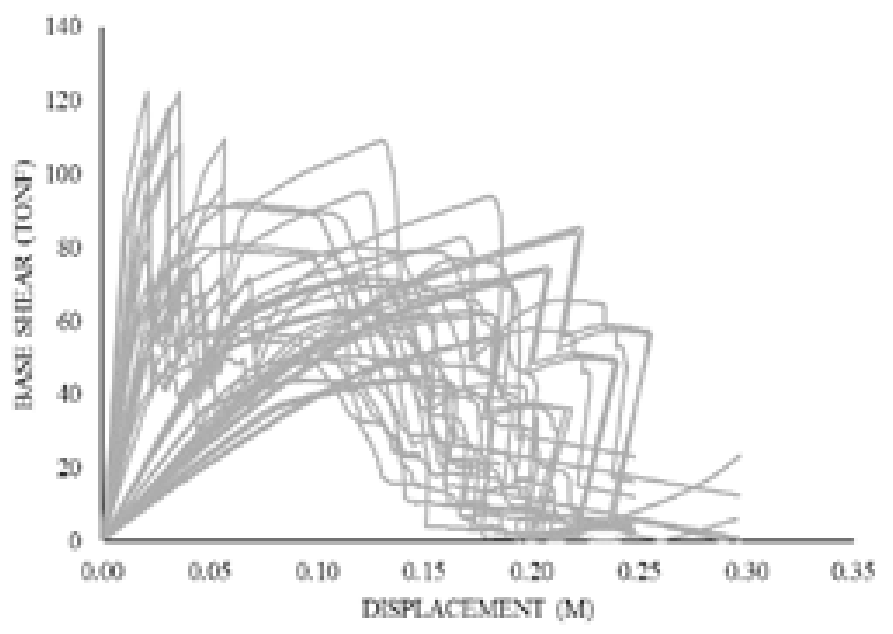

Figura 22. Curvas de Capacidad, Tipología 2, Modelos 1 - 36. № Corregidas, estabilidad numérica. 
En la Figura 23, se observa las cur- máticos que representan la variabilidad vas de capacidad de los modelos mate- de la tipología 3.

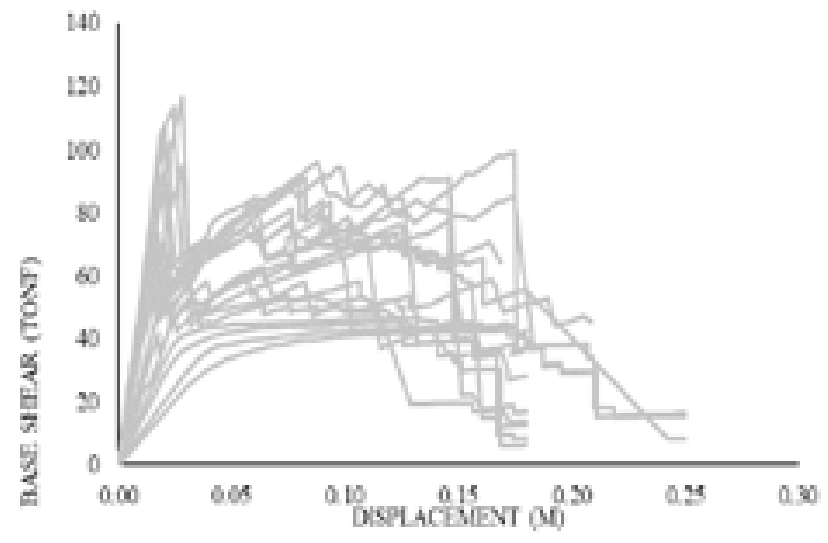

Figura 23. Curvas de Capacidad, Tipología 3, Modelos 1 - 20. № Corregidas, estabilidad numérica.

En la Figura 24, se observan las matemáticos que representan la variabicurvas de capacidad de los modelos lidad de la tipología 4.

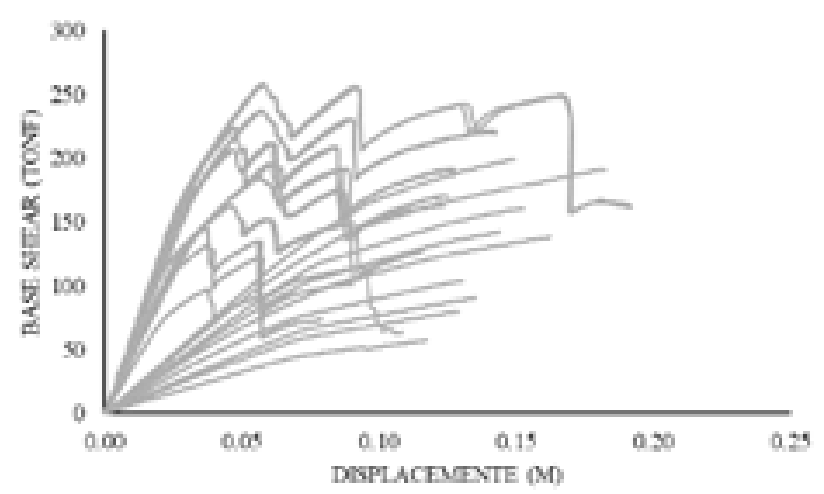

Figura 24. Curvas de Capacidad, Tipología 4, Modelos 1 - 20. № Corregidas, estabilidad numérica. 
Con base en las curvas de capacidad que describen el comportamiento plástico de las distintas tipologías, se procede a calcular el punto de desempeño teórico en el cual los modelos alcanzan la demanda sísmica teórica para 100 y 475 años de período de retorno.

La demanda sísmica está representada por cuatro escenarios con base en la curva de peligro sísmico y distintos tipos de suelo para la ciudad de Quito.
Para esta investigación la curva de peligro sísmico (Figura 25), se obtiene del NEC15 [6]; de la cual los escenarios sísmicos empleados son los eventos de 475 años y 100 años de período de retorno. El escenario de 475 años de período de retorno es el evento sísmico de demanda para la filosofía de diseño sismorresistente ecuatoriana y 100 años de período de retorno es uno de los eventos sísmicos más probables dentro de la sismicidad histórica Quiteña.

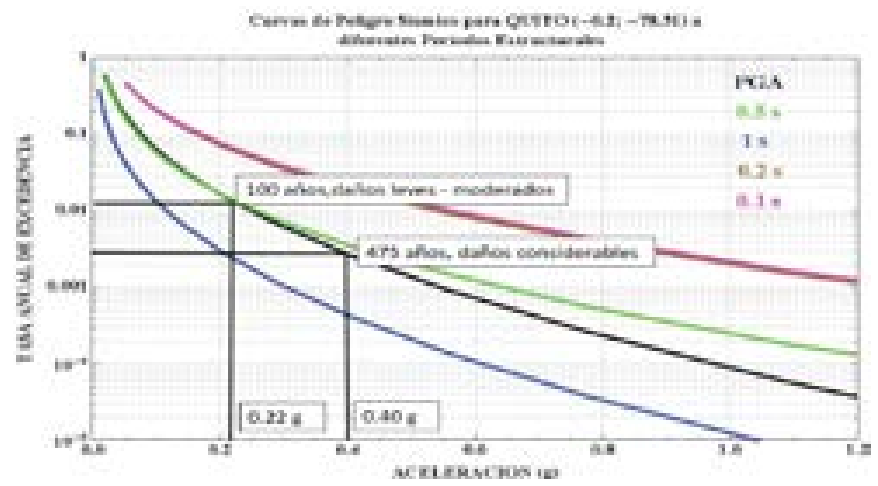

Figura 25. Curva de Peligro Sísmico, Ciudad de Quito, Ecuador (PGA).

Cabe recalcar que el presente análisis de escenarios sísmicos es solo un primer vistazo de la demanda sísmica que se analizará en la segunda parte de esta investigación (Seismic Vulnerability of Quito Ecuador, Phase Two: Fragility and Vulnerability Curves of Structural Typologies, Project GEM-SARA). Dicha in- vestigación incluirá curvas de fragilidad para escenarios de peligrosidad sísmica espectral desde el $2 \%$ de probabilidad de excedencia hasta el $92 \%$ con amplificación de los cinco tipos de suelos descritos en NEC15 [6] mediante métodos estáticos directos y correlación HAZUS [22]. 
Tabla 2. Escenarios Sísmicos.

\begin{tabular}{cccc}
\hline $\begin{array}{c}\text { Escenarios } \\
\text { Sísmicos }\end{array}$ & $\begin{array}{c}\text { Periodo de } \\
\text { Retorno }\end{array}$ & $\begin{array}{c}\text { Suelo } \\
\text { Tipo B }\end{array}$ & $\begin{array}{c}\text { Suelo } \\
\text { Tipo C }\end{array}$ \\
\hline 1 & 475 años & & $\mathrm{X}$ \\
2 & 475 años & $\mathrm{X}$ & \\
3 & 100 años & & $\mathrm{X}$ \\
4 & 100 años & $\mathrm{X}$ & \\
\hline
\end{tabular}

Con base en los escenarios sísmicos de la tabla 2, se calcula el punto de desempeño para cada escenario en función de cada modelo. El punto de desempeño se calcula con base en el procedimiento A del ATC40 [7], considerando la trasformación de los escenarios sísmicos a formato ADRS y las curvas de capacidad a espectros de capacidad.

Tabla 3. Mínimos valores permitidos para SRa and SRv.

\begin{tabular}{lcc}
\hline $\begin{array}{l}\text { Tipo de Comportamiento } \\
\text { Estructural }\end{array}$ & SRa & SRv \\
\hline Tipo A & 0.33 & 0.50 \\
Tipo B & 0.44 & 0.56 \\
Tipo C & 0.56 & 0.67 \\
\hline
\end{tabular}

Para prevenir una reducción excesiva del espectro ADRS por estabilidad numérica se emplea el FEMA273 [8]. Debido a la variabilidad de las tipologías, se considera un comportamiento estructural tipo B para la máxima reducción permisible del espectro ADRS. 
A continuación, se muestra algu- empeño resultado del algoritmo. nos de los resultados de puntos de des-

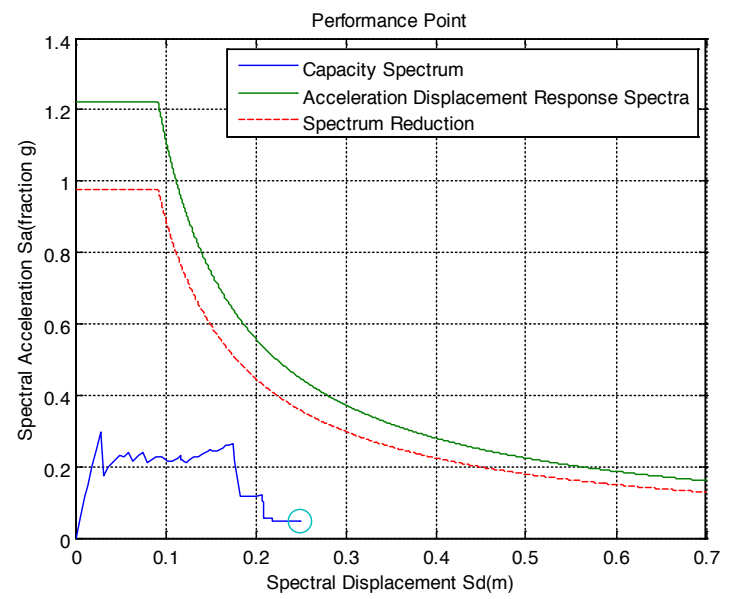

Figura 26. Punto de desempeño, Tipología 3 - Modelo 16, escenario sísmico 1.

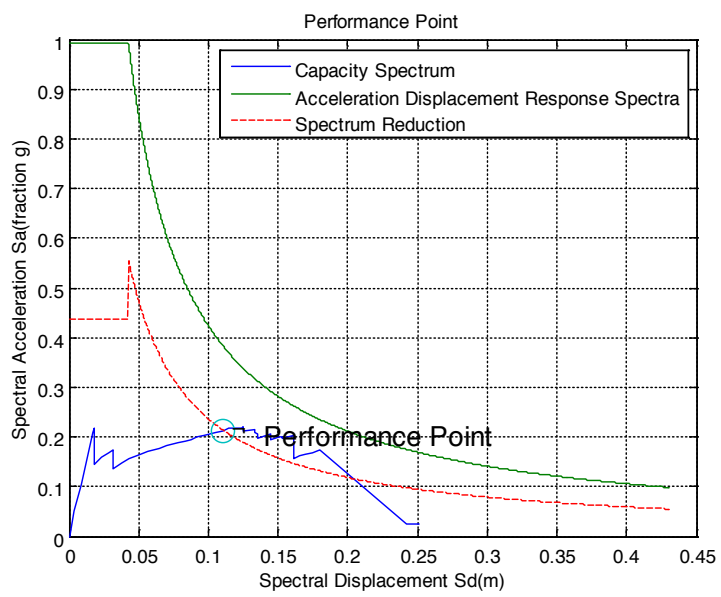

Figura 27. Punto de desempeño, Tipología 3 - Modelo 11, escenario sísmico 2. 


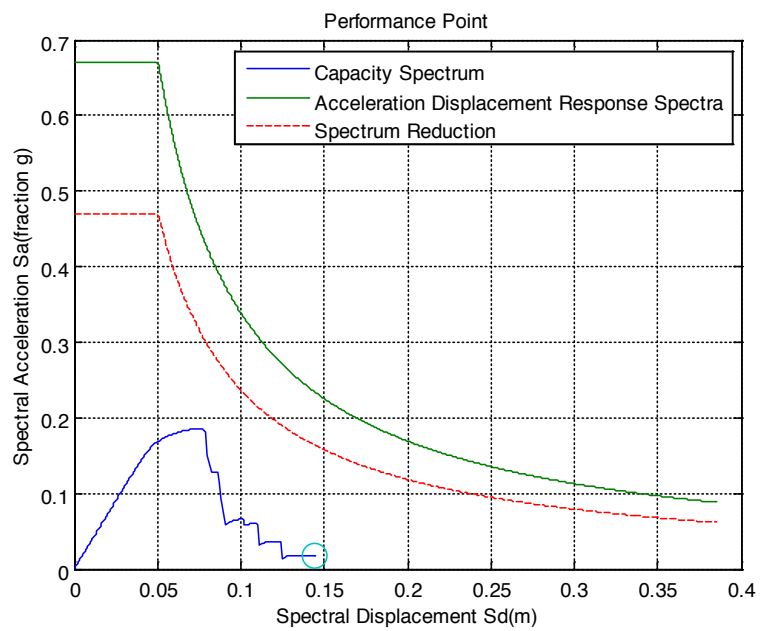

Figura 28. Punto de desempeño, Tipología 2 - Modelo 19, escenario sísmico 3.

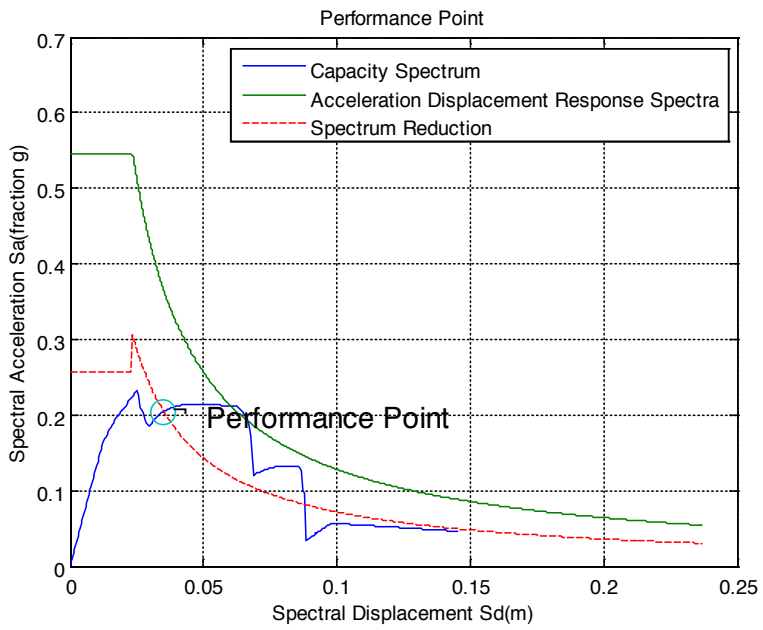

Figura 29. Punto de desempeño, Tipología 2 - Modelo 11, escenario sísmico 4. 
Tabulados los resultados del punto de desempeño para cada modelo representativo de las distintas tipologías se obtuvieron los siguientes resultados (siendo estos solo un primer vistazo a la fragilidad intrínseca de cada tipología la cual se verá en detalle en la fase dos de la presente investigación):

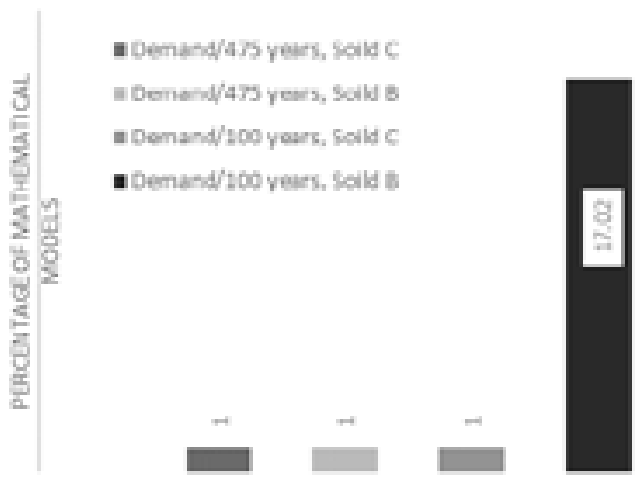

Figura 30. Porcentaje de modelos matemáticos, que alcanzan la demanda (Tipología 1), todos los escenarios sísmicos.

Similar proceso se resume en las respectivamente.

Figuras 31 y 32, para las tipologías 2 y 3 


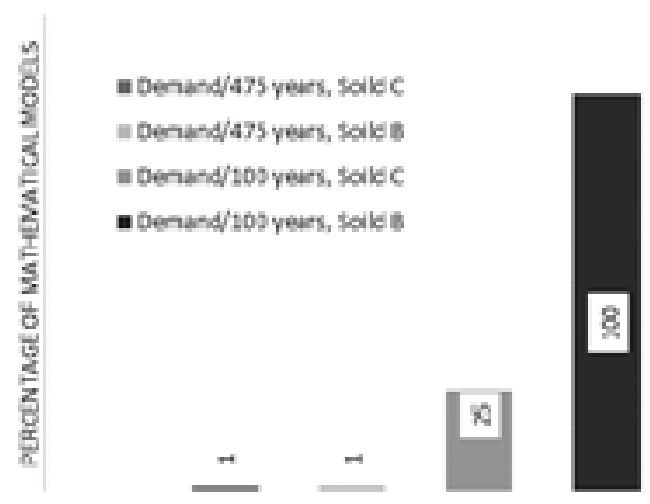

Figura 31. Porcentaje de modelos matemáticos, que alcanzan la demanda (Tipología 2), todos los escenarios sísmicos.

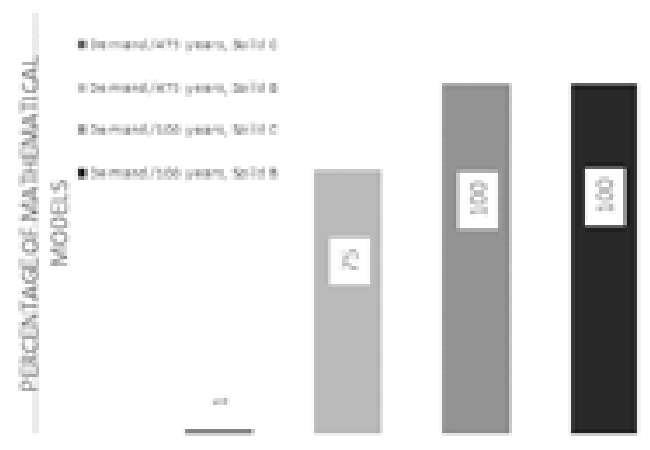

Figura 32. Porcentaje de modelos matemáticos, que alcanzan la demanda (Tipología 3), todos los escenarios sísmicos. 


\section{COLAPSO LOCAL VERSUS}

\section{ESTABILIDAD NUMÉRICA}

Como se menciona en el numeral 3.1, las curvas de capacidad se calculan hasta encontrar la estabilidad numérica o hasta alcanzar un desplazamiento en el punto de control igual al $5 \%$ de la altura total de la edificación. Debido a que en la modelación matemática de cada tipología, se incluye el efecto no lineal-frágil de las mamposterías; los pórticos presentan una rápida plastificación CP una vez que se presenta la falla de las mamposterías. Esto se debe a la pobre relación de rigideces entre los elementos estructurales y no estructurales y las vulnerabilidades intrínsecas de cada tipología. Es por este motivo que se limitan las curvas de capacidad hasta encontrar el primer colapso local de un pórtico dentro de la estructura tridimensional.

Se considera el colapso local (fragilidad local) de un pórtico cuando se dé una rotulación "CP" o "D" en la base de al menos el $75 \%$ de las columnas del primer piso. Con base en lo expuesto en los párrafos anteriores, se recalcula el porcentaje de modelos matemáticos que alcanzan la demanda sísmica.

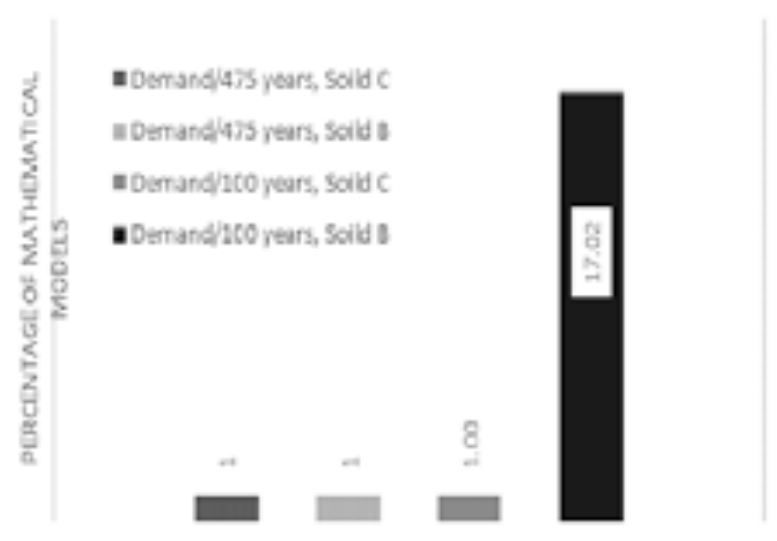

Figura 33. Porcentaje de modelos matemáticos, que alcanzan la demanda (Tipología 1), todos los escenarios sísmicos, limitado por fragilidad local. 


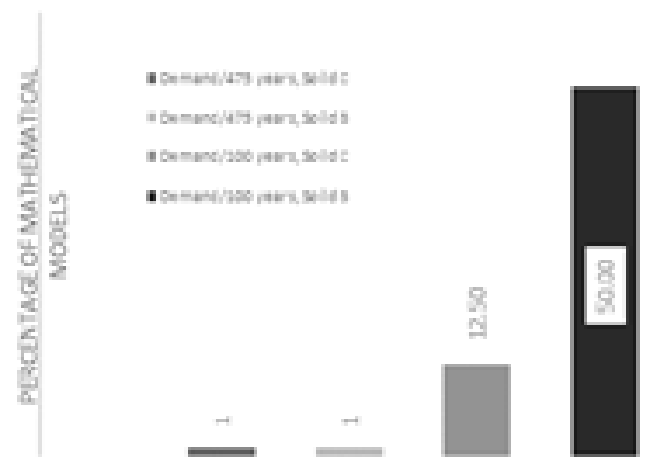

Figura 34. Porcentaje de modelos matemáticos, que alcanzan la demanda (Tipología 2), todos los escenarios sísmicos, limitado por fragilidad local.
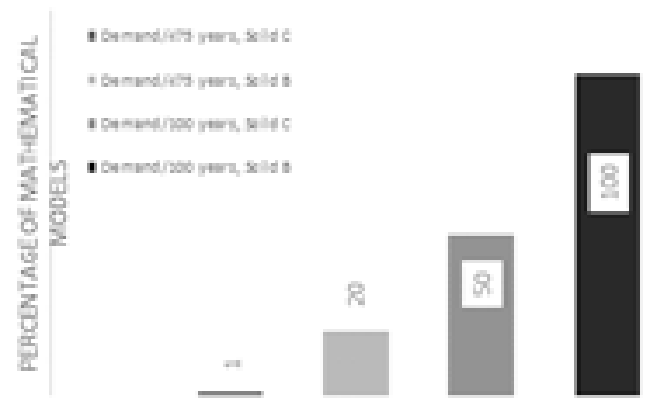

Figura 35. Porcentaje de modelos matemáticos, que alcanzan la demanda (Tipología 3), todos los escenarios sísmicos, limitado por fragilidad local.

\section{COSTO DE REPOSICIÓN}

El costo de reposición expresado en esta publicación es una primera aproximación, del valor en dólares de reconstrucción de las tipologías analizadas, debido al colapso estructural frente a los escenarios sísmicos antes presentados. Es importante hacer notar que el presente costo de reposición se abracara a profundidad en la tercera parte de la presente investigación. (Seismic Vulnerability of Quito, Ecuador, Phase Three: 
Exposure Model of Structural Typologies, Project GEM-SARA).

Se ha considerado el costo de forma aproximada por $\mathrm{m}^{2}$ extrapolado al costo por unidad habitacional multifamiliar. Se a considerando adicionalmente a los cuatro escenarios sísmicos, tres escenarios que contemplan el grado de confiabilidad del cálculo realizado y distintos costos por unidad de vivienda.

El costo referencial no contempla daños a infraestructura, eléctrica, sani- taria, potable, vial, costo cesante de actividad económica del sector donde se emplazan las tipologías.

En la tabla 4, se presentan los tres escenarios adicionales para obtener el costo de reposición de la tipología 4; cada tipología posee sus propios niveles de confiabilidad del cálculo y costos de reposición.

Tabla 4. Valores de confiabilidad del cálculo/costo de reposición, Tipología 3.

\begin{tabular}{lccc}
\hline $\begin{array}{l}\text { Probabilistic } \\
\text { Conditions }\end{array}$ & $\begin{array}{c}\text { Confidence level of } \\
\text { the calculation }\end{array}$ & $\begin{array}{c}\text { Cost of replacement } \\
\text { per m² }^{2}\end{array}$ & $\begin{array}{c}\text { Cost of replacement } \\
\text { per unit }\end{array}$ \\
\hline Best & $60 \%$ & 150 usd & 43200 usd \\
Most Likely & $80 \%$ & 400 usd & 115200 usd \\
Worst & $90 \%$ & 600 usd & 172800 usd \\
\hline
\end{tabular}

Para cada tipología se realiza el cálculo global del costo de reposición del porcentaje de modelos matemáticos que no alcanzan los distintos niveles de demanda sísmica.

En la Figura 36 se observa un resumen de cálculo del mejor escenario propuesto en la Tabla 4, para los distintos escenarios sísmicos aplicados a la tipología 4. Similar proceso se realiza para cada tipología considerando todos los escenarios propuestos. 


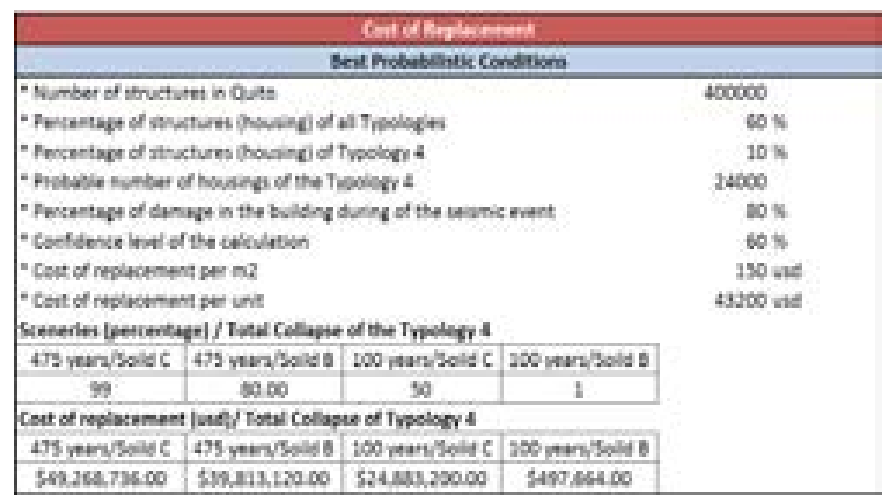

Figura 36. Costo de reposición, Mejor escenario probabilístico.

Tabulando los resultados de las 3 tipologías analizadas se tiene el siguiente cuadro de costos de reposición aproximada.

Para conocimiento del lector; en paralelo a la presente investigación estamos realizando el modelo de exposición de la muestra analizada con base en los datos generados en el censo de población y vivienda del año 2010 y un análisis profundo sobre el costo estructural de las unidades estructurales uni y multifamiliares del DMQ. Datos necesarios para la modelación del riesgo.

Tabla 4. Costo de Reposición aproximado.

\begin{tabular}{cll}
\hline Probabilistic Conditions & Cost of replacement (usd) & Seismic Scenaries \\
\hline \multirow{3}{*}{ Best } & $\$ 128,144,333.00$ & 475 years/Soild C \\
& $\$ 118,688,717.00$ & 475 years/Soild B \\
& $\$ 96,318,720.00$ & 100 years/Soild C \\
& $\$ 46,122,547.00$ & 100 years/Soild B \\
& $\$ 424,805,990.00$ & 475 years/Soild C \\
Most Likely & $\$ 386,983,526.00$ & 475 years/Soild B \\
& $\$ 304,943,616.00$ & 100 years/Soild C \\
& $\$ 131,287,450.00$ & 100 years/Soild B \\
& $\$ 825,422,400.00$ & 475 years/Soild C \\
& $\$ 754,505,280.00$ & 475 years/Soild B \\
Worst & $\$ 596,030,400.00$ & 100 years/Soild C \\
& $\$ 257,313,600.00$ & 100 years/Soild B \\
\hline
\end{tabular}




\section{CONCLUSIONES}

El comportamiento de las distintas unidades estructurales (provenientes de la muestra) indiferentemente de la tipología, presenta una falla frágil en la base de las columnas una vez que las mamposterías colapsan ante la solicitación sísmica. Este fenómeno se produce debido a la baja relación rigideces entre vigas y columnas, la deficiente capacidad a cortante de las secciones y la mala distribución de mamposterías dentro de la edificación.

De los resultados expuestos en la Figura 30, se observa que la tipología con mayor grado de fragilidad frente a los distintos escenarios sísmicos es la tipología 1, la cual corresponde al $16 \%$ de las edificaciones uni y multifamiliares del DMQ.

Es alarmante observar que en todas las tipologías frente al escenario sísmico de 475 años de período de retorno amplificado por un suelo tipo C; más del $80 \%$ de los modelos matemáticos no alcanzan la demanda sísmica; es decir más del $80 \%$ de las combinaciones tipológicas frente a este escenario sísmico podrían ser una pérdida total económica no implicando necesariamente un colapso total estructural.

De las diferentes referencias bibliográficas sobre el comportamiento no lineal-frágil de la mampostería, el modelo propuesto Reinhorn obtiene resultados que se aproximan mejor a los ensayos experimentales obtenidos por Alomoto.

El modelo propuesto por Asteris se emplea en la tipología 2 para representar las aberturas en mamposterías, sin embargo, no se tienen ensayos experimentales para calibrar dicho comportamiento.

Para la distribución de carga lateral en el método NSP se considera una variación lineal, una variación proporcional a la forma del primer modo de vibración y una variación según la superposición modal; encontrando que forma de distribución varia muy poco entre cada metodología.

Es importante observar que en el presente manuscrito aún no se realiza el cálculo de fragilidad ni vulnerabilidad. Pues dichos análisis se realizarán en la siguiente parte de la investigación "Seismic Vulnerability of Quito Ecuador, Phase Two: Fragility and Vulnerability Curves of Structural Typologies, Project GEM-SARA".

Dicho análisis requiere conocer la amenaza sísmica al menos a nivel de tasa anual de excedencia con una confiabilidad aceptable; lo cual en este momento en nuestro medio aún está en debate 
debido a la discrepancia que existe entre los distintos modelos de microzonificación para el DMQ.

El lector debe considerar que los resultados aquí expuestos son el primer vistazo de los resultados definitivos que se expondrán en la tercera fase de la investigación. Y que, si bien el rigor matemático empleado en la generación de las curvas de capacidad presenta resultados validos y contrastados, los efectos sobre el modelo de riesgo se podrán ver afectados debido a la profundidad y aceptación de los modelos de amenaza sísmica y número de estructuras relevadas en campo.

No con esto se quiere decir que la fragilidad intrínseca de cada estructura variara significativamente, sino que el riesgo puede verse afectado en una medida que puede o no ser considerable según la confiabilidad de la amenaza. 


\section{BIBLIOGRAFÍA}

Alomoto [2015], Resultados de ensayos experimentales de pórtico rellenos con mampostería, Centro de Investigación de la Vivienda.

Asteris P.G., Cavaleri L., Di Trapani F., Sarhosis V. [2015] A macro-modelling approach for the analysis of infilled frame structures considering the effects of openings and vertical loads.

ASCE 41-06, 2006,Seismic Rehabilitation of Existing Buildings, American Society of Civil Engineers, Reston, Virginia

ATC 40. Seismic Evaluation and Retrofit of Concrete Buildings, California Seismic Safety Commission, Volume 1, Report No SSC 96 -01, California, 1996

Ayala C. Almazán J. (2015). "Modelo Celosía No-Lineal para muros de albañilería armada de bloque con relleno parcial". XI Congreso Chileno de Sismología e Ingeniería Sísmica, paper № 195.

Blandon, C.A. [2005] Implementation of an Infill Masonry Model for Seismic Assessment of Existing Buildings, Individual Study, European School for Advanced Studies in Reduction of Seismic Risk (ROSE School), Pavia, Italy.
Crisafulli F.J. [1997] Seismic Behaviour of Reinforced Concrete Structures with Masonry Infills, PhD Thesis, University of Canterbury, New Zealand.

FEMA 273. NEHRP Guidelines for the Seismic Rehabilitation of Buldings, Federal Emergency Management Agency, Washington.D.C, 1997.

Hazus - MH 2.1. Advance Engineering Building Module (AEBM), Washington.D.C, 2001.

Karsan, I.D. and Jirsa, J.O. (1969). "Behavior of concrete under compressive loading", Journal of Structural Division, ASCE, Vol. 95, No. 12, pp. 2543-2563.

Mander Mander, J. B., Priestley, M. J. N. and R. Park, "Theoritical Stress - Strain Model for Confined Concrete, Journal of Structural Engineering", Journal of Structural Engineering, Vol. 114, No 8, pp 22686, 1988.

Matlab, Language of Techical Computing, MathWorks, U.S.,2000.

NEC-15, Norma Ecuatoriana de la construcción, Editorial Dirección de comunicación Social MIDUVI, 2014,ISBN:000000.

Opensees, Open System for Earthquake Simulation, Pacific Earthquake Engineering Research Center, 
University of California, 1999.

Panagiotou, M., (2012), "Nonlinear cyclic truss Model for reinforced Concrete Walls", ACI Structural Journal, Technical Paper: 205-214.

Perform 3D [2011]

Popovics, S., "A numerical approach to the complete stress strain curve for concrete". Cement and concrete research, 3(5), 583-599, 1973.

Reinhorn A.M., Madan A., Valles R.E., Reichmann Y., Mander J.B. [1995] Modeling of masonry infill panels for structural analysis.

Seismosoft [2016] "SeismoStruct 2016 - A computer program for static and dynamic nonlinear analysis of framedstructures," available from http://www.seismosoft.com.
Smyrou E., Blandon C.A., Antoniou S., Pinho R., Crisafulli F. [2011] "Implementation and verification of a masonry panel model for nonlinear dynamic analysis of infilled RC frames," Bullettin of Earthquake Engineering, DOI 10.1007/ s10518-011-9262-6.

Sap2000, Static and Dynamic Finite Elements Analisys of Structures Advance, Computer and Structures, Inc. 1995 University Ave. Berkeley, CA 94704, 1995.

Williams, S.A., (2013), "Numerical Analysis of Reinforced Masonry Shear Walls Using the Nonlinear Truss Approach", Thesis Master of Science in Civil Engineering, Virginia Polytechnic Institute and State University 
ill|| 80

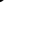

